\title{
EDITORIAL
}

\section{Does Telepathy Threaten Mental Privacy?}

\section{Stephen E. Braude}

https://doi.org/10.31275/20201829

Creative Commons License CC-BY-NC

\begin{abstract}
A long-standing concern (or at least a belief) Aabout ESP, held by both skeptics and believers in the paranormal, is that if telepathy really occurs, then it might pose a threat to mental privacy. And it's easy enough to see what motivates that view. Presumably we like to think that we enjoy privileged
\end{abstract} access to our own mental states. But if others

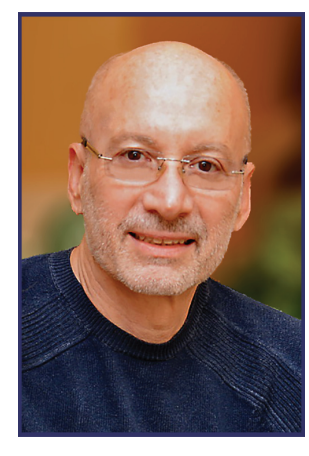
could come to know telepathically what we're thinking or feeling, then (among other disquieting prospects) that would mean that our sins of the heart and most embarrassing or repulsive fleeting thoughts would potentially be available for public inspection.

But how well-founded is that belief or concern? To get a grip on the issues, we should begin by considering the valuable distinction (perhaps first mentioned by C. D. Broad [Broad, 1953, 1962] between telepathic (or clairvoyant) cognition and telepathic (or clairvoyant) interaction. As you would expect, every instance of the former would be an instance of the latter, but the converse doesn't hold-that is, ESP interaction may occur without ESP cognition. To see why this matters, we must take a closer look.

If telepathic cognition occurs at all, it would presumably be a form of non-sensorial knowledge about another individual's state of mind. More specifically, it would be a state of affairs in which so-called "percipient" A comes to know something about a telepathic interaction $A$ has with another individual $B$. And what kind of things might $A$ telepathically come to know? Well, presumably, in its most robust (and most intrusively intimidating) form, $A$ would learn what's going on in $B$ 's mind-that is, that $B$ is having certain thoughts, perceptions, or emotions. But it would still be an instance of telepathic cognition- 
admittedly, less intimidating or threatening to one's mental privacy-if $A$ learned merely that $B$ was the telepathic cause of $A$ 's current thought or experience-that is, that $B$ was directly influencing or interfering with A's stream of consciousness, whether or not A's resulting thoughts or experiences were those of $B$ or known by $A$ to be those of $B$.

However, Broad shrewdly recognized that the evidence for telepathy was seldom (if ever) evidence of these kinds of knowledge. On the contrary, what we usually find is evidence suggesting only telepathic interaction. In what is probably telepathy's most commonly reported form, person B's mental state merely influences that of $A$, and $A$ learns nothing from the process about $B$ 's causal role, much less details of what $B$ is thinking or feeling. For example, it would be telepathic interaction (not cognition) if my thought of the Eiffel Tower directly (that is, without sensory mediation) caused a remote person simply to think about the Eiffel Tower (or about towers generally, or about the Tower of London) — that is, without that person realizing that I played a causal role in that event, much less that I was thinking about the Eiffel Tower. Similarly, it would be a case of clairvoyant interaction (not cognition) if a burning house was the direct cause of someone at a remote location simply thinking about fire (or heat), or feeling a need to apply aloe to one's skin, or having the urge to watch Blazing Inferno. There's no need (and arguably not even a temptation) in these cases to insist that the percipient knows (presumably subconsciously) what caused the experience in question. The telepathic and clairvoyant scenarios would simply be paranormal analogues to the way our bombardment with environmental information can trigger various thoughts and associations, and perhaps distant or idiosyncratic associations at that. In both the paranormal and normal cases, we may be oblivious to the causal processes that led to our thoughts.

As it happens, when we look closely at the evidence for apparent telepathy, it does indeed seem as if it's largely (though perhaps not entirely) evidence merely of telepathic interaction. But we must make an important admission even before we look at the evidence-namely, that as far as we know, telepathy could occur between strangers or only very casual acquaintances, with the percipients never learning why, or even that, they had experienced telepathically influenced (or tainted) mental states. We have no grounds at present for denying this 
possibility, and, if that sort of telepathy occurs, we have no idea whether those moments of telepathic interaction are frequent or rare.

Granted, percipients in spontaneous cases (such as crisis cases) sometimes seem to know (or at least infer or suspect) who caused the surprising or anomalous thought they just had. And that's to be expected. After all, if I have an intrusive thought that my friend Jones had an accident and is in pain, it's a natural (though rationally risky) next move to infer that l'd been in touch psychically and momentarily with Jones. Nevertheless, it's often (if not usually) the case that percipients only learn some time after their experience, and through normal channels of information, that their anomalous mental states corresponded to the roughly contemporaneous thoughts or experiences of a remote individual in crisis. So in those cases at least, knowledge of that correspondence doesn't seem to be telepathic cognition.

In the interest of full disclosure, I must report that one could attempt here a theoretical maneuver that perhaps only a philosopher could love. The point of the maneuver is to argue that even in cases where there seems to be only mere telepathic interaction, what we find instead is a cornucopia of cognition. One could argue that the percipient's original telepathically caused mental state was indeed telepathic cognitionpresumably subconscious. And then one could claim that the percipient's subsequent knowledge of the correspondence between the earlier telepathic experience and the agent's crisis is a form of second-order knowledge - that is, non-telepathic knowledge that the earlier mental state was an instance of telepathic knowledge. So one could claim that at the time of the original telepathic interaction, the percipient knows that Jones is (or was) in crisis but doesn't know that (s)he knows this. ${ }^{1}$

However, if the appeal to second-order knowledge is viable at all, it may be applicable only to crisis cases. More typically, correspondences between the thoughts of agent and percipient are less clear-cut, and don't seem at all to refer or point to a presumptive agent. So they don't require positing any telepathic awareness or cognition of the agent's causal role, much less what the agent's mental state is. For example, in one well-known experiment in ostensible dream telepathy, the agent was concentrating on a target-print of Bichitir's Man with Arrows and Companion, which portrays three men in India sitting outdoors. One holds a musical stringed instrument; the most prominent of the three 
holds a bow and arrows. The third man has a stick over his shoulder that looks like a rifle muzzle. One minor detail of the painting is a stake with a rope tied around it, and the percipient seemed to pick up on that small detail and incorporate images of rope prominently in his dreams. He had five dreams that night, and three of them contained rope (or coiled rope) as a prominent feature. Moreover, in another dream the percipient saw a "hammock in which there was an awful lot of suspended strings" (Ullman, Krippner, \& Vaughan, 2002, p. 125).

In another study, the agent (an orthodox Jew) concentrated on a print of Chagall's The Yellow Rabbi, in which an old rabbi sits at a table with a book in front of him. The subject of the experiment was a Protestant. In one dream, he saw a man in his 6os riding in a car. In another he reported "a feeling of older people. The name of Saint Paul came into my mind." In another, he dreamt of a professor of humanities and philosophy reading a book. In the summary of his dreams the next morning, the subject reported, "So far, all I can say is that there is a feeling of older people. ... The professor is an old man. He smoked a pipe, taught humanities as well as philosophy. He was Anglican minister or priest" (Ullman et al., 2002, pp. 91ff).

So when percipients are participating in informal experiments with friends or in more formal experiments (like the Ganzfeld), it may not be outrageous to say that they can know whose mental state affected their own. But if so, it's only because the percipients understand from the start, and through normal channels of information, that there's a designated agent (or "sender") and that the goal of the experiment is to find significant correspondences between the mental states of the agent and percipient. There doesn't seem to be even a superficial basis for saying that percipients had telepathic knowledge all along, but didn't know that they know.

In any case, there's another reason to question whether the percipients' conjectures in these situations are types of knowledge. Perhaps the following analogy will make this clear. Suppose an unidentified person surreptitiously deposits a message with my signature or photo on your doorstep. Obviously, the deposited object doesn't indicate unambiguously who put it there. After all, it could be left there mischievously by someone other than me. Knowledge of the object's source can't be derived simply from the object's presence 
on your doorstep. Similarly, a percipient's telepathically induced state won't point unambiguously to its source, even if it contains features that seem to "refer" or point to a source.

Besides, and as the dream-telepathy examples illustrate, a telepathically induced state needn't contain any such clues or pointers, and the vast majority of ostensible telepathic interactions lack those features. So there's no reason to think that paranormal experiences must include (or be preceded by) a warning or marker-something analogous to a flourish of trumpets, announcing that the experience is paranormal. Therefore, as long as percipients lack additional, normally acquired contextual information about the presumed origin of their ESP-induced mental state, that state might seem to be a merely random intrusive thought-that is, one of the occasional incongruous or unexpected, and easily ignored, mental states probably all of us have during the course of the day.

We should now see clearly one reason why the ESP cognition/ interaction distinction matters. If (as it seems) most ostensible telepathy cases are examples of telepathic interaction but not telepathic cognition, then we may have no grounds for worrying about an ongoing (or at least significant) loss of mental privacy. And that may be enough for us to feel we're at least generally off the hook, and that we'll be able to shield our most reprehensible or embarrassing thoughts from prying minds.

Incidentally, this is one reason why the lamentably trendy practice of replacing the venerable terms "ESP," "telepathy," and "clairvoyance" with the catch-all term "anomalous cognition" (AC) is egregiously wrong-headed. (See, e.g., May, Spottiswoode, Utts, \& James, 1995; May, Utts, \& Spottiswoode, 1995a, 1995b.) I criticized that practice some time ago (Braude, 1998), and I've refined and expanded that critique in a forthcoming book (Braude, 2020). The same may be said about the even more recently trendy (and arguably incoherent) terms "nonlocal awareness" or "nonlocal consciousness." But what I hope the preceding has shown is that by ignoring the useful cognition/interaction distinction, those terminological reduction strategies fail to supply the taxonomic resources even for beginning to describe adequately the relevant, interesting, and empirically unresolved issues discussed above.

Let's pursue the matter a bit, because it should shed further light on the feasibility of positing telepathic cognition. Perhaps the clearest 
examples of mere telepathic interaction are those in which a person's mental states seem to be the direct cause of a remote individual's actions. As Jule Eisenbud noted,

That a person can mentally influence not just the thoughts of other persons extrasensorially at a distance but also their decisions and actions must be one of the oldest facts of nature known to man. It has been woven into the core of every primitive culture described by anthropologists. (Eisenbud, 1992, p. 87)

But the evidence for this isn't simply anecdotal. In relatively modern times, the phenomenon has been investigated systematically and experimentally, and the best-documented cases concern the induction of hypnotic states at a distance. For example, hypnosis at a distance was reported in the eighteenth century by the early mesmerists, including Puységur, and then, in the mid to late nineteenth century, in studies by Janet and Richet (Janet, 1885, 1886; Richet, 1885, 1888; for more details, see Eisenbud, 1992). Perhaps defenders of the use of the term "anomalous cognition" forgot about this body of evidence, or more likely didn't know about it at all. Too often, psi researchers enter the field with—at best-only a very superficial knowledge of the rich history of relevant empirical and theoretical work that preceded them. ${ }^{2}$ Nevertheless, it seems indefensible for partisans of the new terminology to exclude the phenomenon from their terminological considerations.

Interestingly, though, when it comes to the studies of apparent telepathic mind-control, even those familiar with the evidence do their best to avoid the subject. For example-and despite their successesJanetand Richetabandoned the studyofhypnosis shortlyaftercompleting their experiments and retreated to less momentously intriguing lines of investigation. Moreover, when Vasiliev demonstrated hypnosis at a distance once again in the mid twentieth century, the community of psi researchers (and of course the rest of the academy) failed to pursue the matter further. ${ }^{3}$ In fact, and contrary to what usually happens when parapsychologists report much less dramatic and noteworthy effects, there was no flurry of replication attempts-actually, no attempts at all. It's not that Vasiliev's work (or that of his precursors) was poorly done. Rather, it seems clear that the phenomenon was simply terrifying in its 
implications and thus too easily ignored.

Partisans of the term "anomalous cognition" might be tempted to argue that the expression "cognition" was merely a terminological infelicity, suggesting (admittedly misleadingly) that every instance of $A C$ is a kind of knowing or cognition. What matters, they might say, is that AC is merely a kind of anomalous "information transfer" or "acquisition of information." So they might suggest that some sort of information is acquired or transferred even when a thought about the Eiffel Tower causes someone to think about the Tower of London, or when a burning house causes someone to think about matches, or when someone remotely responds to my hypnotic command to fall asleep. But even if that terminological maneuver works for some instances of ESP interaction, other ostensibly telepathic and clairvoyant interactions more clearly resist definition or analysis in terms of information transfer.

At stake here is another intimidating issue, a modest extension of what we considered in connection with hypnosis at a distancenamely, that telepathic influence could-at least theoreticallybe used for total control of another person's mind and body. Now presumably we wouldn't want to say that telepathic dominion over my thoughts and actions can be understood in terms of transfer or acquisition of information. After all, we wouldn't invoke information transfer to explain extreme, but normal, forms of forcing another to act. Whatever exactly the process might be, it's not analogous (say) to understanding and responding to a command. The clearest examples are probably ordinary cases of behavioral coercion. It's not information transfer, in any helpful epistemic sense of the term "information," if I physically overpower you and compel you to pull the trigger of a gun, and we similarly wouldn't consider it to be information transfer if my willing alone both prevented you from exercising your volition and also compelled you to fire the gun. Perhaps we should describe that telepathic version of coercion as a form of possession. But what matters is that the degree of control posited in these coercion scenarios resembles the control of a puppet, and it's thoroughly unilluminating to describe the puppeteer as transferring information to the puppet. Likewise, we wouldn't consider it to be information transfer if I telepathically seized control of your mental life, blocking your access to 
your own stream of consciousness, and forcing you to have thoughts that are not your own. Victims of such telepathic influence would have no awareness at all—much less knowledge—of the interaction. And that's one reason why we wouldn't hold them morally responsible for their thoughts and actions at the time.

Before you dismiss these proposed scenarios as mere fantasy, we should note that there's actually an empirical basis for concern about this issue. It's not simply an abstract, theoretical matter we can acknowledge and then conveniently put out of mind. Total telepathic control of a human organism is ostensibly what happens during mediumistic trance-impersonation, in which the medium's body (and presumably, brain) are controlled by a deceased communicator who also apparently displaces the medium's waking consciousness. This is the process F. W. H. Myers called "telergy," and it remains an open question whether discarnate telepathic control is what really happens during mediumistic trance impersonations, or whether (say) it's the medium's dissociative dramatic personation instead, with occasional verifiable mediumistic ESP thrown in for good measure. ${ }^{4}$ At any rate, if there's a bright side to the possibility of telergy, it's that the process doesn't seem to require or involve some dreaded form of "mindreading" on the part of either agent or percipient. Rather, it would be a situation in which one individual's mental states displace another individual's ordinary stream of consciousness.

Partisans of "anomalous cognition" might be tempted to reply that telergy should properly be called "anomalous perturbation" (AP), or (in more virtuous language) "PK". But that would blur the admittedly somewhat fuzzy, but at least apparently useful, distinction between telepathic influence and PK. For all we know at our still preliminary level of understanding, the paradigmatic PK events of levitating a table, materializing a human figure, or biasing a random event generator, may be significantly different processes, not only from each other but also from directly influencing a person's thoughts or actions. So until we have good reason for claiming that all these phenomena can be similarly explained, it seems unwise at the very least to embrace terminology that prevents us from tentatively classifying the latter only as a distinct, telepathic process.

Actually, conflating telepathic influence and PK will probably appeal only to physicalists who would interpret the latter as a purely 
physical process and the former as a kind of physical influence on the percipient's brain. But if (as it seems) reductive physicalism is generally untenable, it again seems wise (for now at least) to entertain the possibility that telepathic influence and PK are distinct phenomena.

We're fortunate to have developed the linguistic resources for making fine distinctions between classes of phenomena whose differences certainly make sense in theory, and which also seem to have empirical warrant. If later empirical or theoretical advances show that our distinctions have no basis in fact and only apparent theoretical utility, we can then comfortably simplify our arsenal of parapsychological categories. But we're a long way from that point. In the meantime, then, the proposed taxonomical reform of replacing "ESP," "telepathy," and "clairvoyance" with "anomalous cognition" is unacceptably coarse, quite apart from the other serious shortcomings I enumerate elsewhere (Braude, 1998, 2020).

Now we can return to the issue of mental privacy. We've seen that there are quite diverse, and even unsettling, forms of apparent telepathic interaction without cognition. However, it remains an open question whether we have decent evidence of any form of telepathic cognitionespecially of a kind that would justify fearing the loss of privileged access to our own mental states. If this Editorial has helped at all to clarify the issues, then perhaps we now have a better idea what sort of ostensibly telepathic evidence to look for. In the meantime, we can probably and comfortably continue living our unsavory private inner lives.

\section{NOTES}

- We also can't rule out that the percipient's telepathically influenced experience occurs simultaneously with clairvoyant awareness of the crisis occurring to the agent. In that case, we should be reluctant to consider the incident a case purely of telepathic cognition-perhaps GESP [general ESP] cognition instead.

2 This is not entirely their fault. Parapsychology, unlike mainstream disciplines, offers few opportunities to undertake systematic and comprehensive study of psi before embarking on one's own research.

3 Vasiliev (1976). For a good discussion of telepathy at a distance, see Eisenbud (1970, Chapter 5; 1992, Chapter 6).

4 See Braude (2003) for a discussion of these ideas. 


\section{REFERENCES}

Braude, S. E. (1998). Terminological reform in parapsychology: A giant step backwards. Journal of Scientific Exploration, 12(1), 141-150.

Braude, S. E. (2003). Immortal remains: The evidence for life after death. Rowman \& Littlefield.

Braude, S. E. (2020). Dangerous pursuits: Mediumship, mind ex music. Anomalist Books.

Broad, C. D. (1953). Religion, philosophy, and psychical research. Routledge \& Kegan Paul. [Originally published in Philosophy, 24 (1949), 291-309. doi:10.1017/ So031819100007452]

Broad, C. D. (1962). Lectures on psychical research. Routledge \& Kegan Paul. [Reprinted by Routledge, 2011.]

Eisenbud, J. (1970). Psi and psychoanalysis: Studies in the psychoanalysis of psiconditioned behavior. Grune \& Stratton.

Eisenbud, J. (1992). Parapsychology and the unconscious. North Atlantic Books.

Janet, P. (1885). Note sur quelques phenomènes de somnambulisme. Revue Philosophique de la France et de l'Etrangere, 21, 190-198. [1968 translation, Report on some phenomena of somnambulism, Journal of the History of the Behavioral Sciences, 4(2), 124-131.]

Janet, P. (1886). Deuxième note sur le sommeil provoqué à distance et la suggestion mentale pendant l'état somnambulique. Revue Philosophique de la France et de l'Etrangere, 22, 212-223. [1968 translation, Second observation on sleep provoked from a distance and mental suggestion during the somnambulistic state. Journal of the History of the Behavioral Sciences, 4(3), 258-267.]

May, E. C., Spottiswoode, S. J. P., Utts, J. M., \& James, C. L. (1995). Applications of decision augmentation theory. Journal of Parapsychology, 59(3), 221-250.

May, E. C., Utts, J. M., \& Spottiswoode, S. J. P. (1995a). Decision augmentation theory: Applications to the random number generator database." Journal of Scientific Exploration, 9(4), 453-488.

May, E. C., Utts, J. M., \& Spottiswoode, S. J. P. (1995b). Decision Augmentation Theory: Toward a model of anomalous mental phenomena. Journal of Parapsychology, 59, 195-220. https://www.scientificexploration.org/docs/9/ jse_09_4_may.pdf

Richet, C. (1885). Un fait de somnabulisme à distance. Revue Philosophique de la France et de l'Etrangere, 21, 199-200.

Richet, C. (1888). Expériences sur le sommeil à distance. Revue de l'hypnotisme, 2, 225-240.

Ullman, M., Krippner, S., \& Vaughan, A. (2002). Dream telepathy: Experiments in nocturnal extrasensory perception. Hampton Roads.

Vasiliev, L. L. (1976). Experiments in distant influence: Discoveries by Russia's foremost parapsychologist. Dutton. [Reprinted in 2002 as Experiments in mental suggestion, Hampton Roads.] 